\title{
Netrin-1 prevents the development of cardiac hypertrophy and heart failure
}

\author{
NAN WANG, YUNSHAN CAO and YAN ZHU \\ Department of Cardiology, The People's Hospital of Gansu, Lanzhou, Gansu 730000, P.R. China
}

Received May 26, 2014; Accepted February 10, 2015

DOI: $10.3892 / \mathrm{mmr} .2016 .4755$

\begin{abstract}
The aim of the present study was to examine whether netrin-1 is involved in the development of cardiac hypertrophy, induced by pressure overload. For this investigation, thoracic transverse aortic constriction (TAC) was performed in mice. A total of 18 mice were divided into three groups $(n=6$ per group): Sham, TAC and TAC + recombinant netrin-1. Neonatal rat cardiomyocytes were stimulated with endothelin-1 (ET-1), and samples were collected to examine the expression levels of netrin-1 by western blot analysis and the mRNA expression of A-type natriuretic peptide by reverse transcription-quantitative polymerase chain reaction. It was found that the expression of netrin-1 was decreased in the TAC mice and in the neonatal rat cardiomyocytes in response to ET-1 stimulation. Netrin-1 eliminated ventricular remodeling, cardiac dysfunction and DNA damage during pressure overload. Furthermore, analysis of the signaling events indicated that netrin-1-mediated protection against cardiac hypertrophy was attributed to interruption of the activation of mitogen-activated protein kinase kinase (MEK) kinase-1 (K1)-dependent MEK-extracellular signal-regulated protein kinase $1 / 2$ (ERK1/2) and c-Jun N-terminal kinase $1 / 2$ (JNK1/2). Therefore, netrin-1 prevented cardiac hypertrophy and heart failure through the negative regulation of the MEKK1-dependent MEK-ERK1/2 and JNK1/2 signaling pathways.
\end{abstract}

\section{Introduction}

Cardiac hypertrophy is associated with several forms of heart disease, including ischemic disease, hypertensive heart disease and valvular stenosis, and is a major risk factor for the development of heart failure and subsequent mortality (1). Despite advances in the treatment of heart failure, it remains one of the leading causes of death in industrialized countries (2).

Correspondence to: Dr Nan Wang, Department of Cardiology, The People's Hospital of Gansu, 160 Donggang West Road, Lanzhou, Gansu 730000, P.R. China

E-mail: nanwanggs@163.com

Key words: netrin-1, cardiac hypertrophy, heart failure, mitogenactivated protein kinase
Therefore, elucidation of the mechanisms underlying the progression of cardiac hypertrophy to heart failure is important to develop effective therapeutic strategies for the treatment of heart failure.

Netrin-1 is a laminin-associated protein and is identified as a neuronal guidance cue, directing axons to its targets during the development of the nervous system (3). Netrin-1 mediates its functions through stimulation of the deleted in colorectal cancer (DCC) family receptors, DCC and neogenin, and the UNC5A, UNC5B, UNC5C and UNC5D UNC5 family receptors (4). In addition to its primary function in neuronal development, the expression of netrin-1 outside the nervous system inhibitsthe migration of leukocytes in vitro and in vivo, and attenuates inflammation-mediated tissue injury (5-7). The administration of netrin-1 to mice suppresses infiltration and inflammation in sepsis, AKI, acute lung injury, peritoneal inflammation and whole body hypoxia (8-13). Previous studies have demonstrated that netrin-1 prevents ischemia/reperfusion-induced myocardial infarction (14-16) and a study by Joseph and Quan indicated netrin-1 as a new therapeutic target in cardiovascular disease (17), however, the role of netrin-1 in cardiac hypertrophy has not been investigated.

In the present study, the role of netrin-1 in the development of cardiac hypertrophy and heart failure was investigated. The expression levels of netrin-1 were decreased in the TAC mice and in neonatal rat cardiomyocytes in response to ET-1 stimulation. In addition, the loss of netrin-1 aggravated foetal gene expressions induced by ET-1 stimulation. By contrast, this increase of gene expression was suppressed by the overexpression of netrin-1, and netrin-1 eliminated ventricular remodeling, cardiac dysfunction and DNA damage during pressure overload. Furthermore, analysis of the signaling events indicated that the netrin-1-mediated protection against cardiac hypertrophy was attributed to interruption of the activation of the mitogen-activated protein kinase (MAPK) kinase (MEK) kinase-1 (K1)-dependent MEK-extracellular signal-regulated protein kinase 1/2 (ERK1/2) and c-Jun N-terminal kinase 1/2 (JNK1/2) signaling pathways.

\section{Materials and methods}

Pressure overload models. A total of 18 male 3 month-old wild-type C57 mice weighing 15-20 g and maintained at $25-30^{\circ} \mathrm{C}$ were obtained from Tongji Medical College (Wuhan, China). The mice were anaesthetized by intraperitoneal 
injection with a mixture of ketamine $(80 \mathrm{mg} / \mathrm{kg} / \mathrm{h})$ and xylazine (8 mg/kg/h; Sigma-Aldrich, St. Louis, MO, USA), intubated, and artificially ventilated, as previously described (18). Pressure overload was then induced by performing thoracic transverse aortic constriction (TAC). A standard lead II electrocardiogram was used for recordings throughout the experiment, and the adequacy of anaesthesia was monitored from the disappearance of the pedal withdrawal reflex. A number of animals were administered via tail vein injection with recombinant netrin-1 (eBioscience, Houston, TX, USA) at a dose of $5 \mu \mathrm{g} /$ mouse every 3 days. Sham surgery involved opening the chest without performing thoracic transverse aortic constriction. The cardiac function was evaluated 4 weeks after TAC or sham-surgery by transthoracic echocardiography, using an FFsonic 8900 (Fukuda Denshi Co., Tokyo, Japan) equipped with a $13 \mathrm{MHz}$ phased-array transducer, under anaesthesia with intraperitoneal pentobarbital sodium ( $35 \mathrm{mg} / \mathrm{kg}$; Sigma-Aldrich). The adequacy of anaesthesia was monitored at all times by assessment of skeletal muscle tone, respiratory rate and rhythm, and response to tail pinch. Left-ventricular (LV) fractional shortening (LVFS) was calculated as [LV end-diastolic diameter (LVEDD) - LV end-systolic diameter) / LVEDD] x 100(\%). The mice in the TAC and sham groups were then sacrificed by intraperitoneal injection of ketamine $(1 \mathrm{~g} / \mathrm{kg})$ and xylazine $(100 \mathrm{mg} / \mathrm{kg})$, and their hearts were rapidly excised. The mRNA levels of Mouse A- and B-type natriuretic peptides (ANP and $\mathrm{BNP}$ ) were determined by reverse transcription-quantitative polymerase chain reaction (RT-qPCR). The present study was approved by the Ethics Committee of the People's Hospital of Gansu (Lanzhou, China).

Cultured neonatal rat cardiomyocytes. Hearts were collected from 1-2-day-old male Sprague Dawley neonatal rat pups weighing 2-3 g (Tongji Medical College), promptly following euthanasia by decapitation. Primary cultures of neonatal rat cardiomyocytes were performed, as described previously $(19,20)$. Following serum starvation, the neonatal rat cardiomyocytes were stimulated with endothelin-1 (ET-1; Sigma-Aldrich), and samples were collected to examine the expression levels of netrin-1 by western blot analysis and the mRNA expression of ANP by qPCR. Netrin-1 siRNA was purchased from Thermo Scientific Dharmacon and was used to transfect the cardiomyocytes using GenomOne-Neo (Ishihara Sangyo Kaisha, Osaka, Japan), according to the manufacturer's instructions. The activities of the ET-1-inducible ANP and BNP promoters were evaluated using a luciferase(luc) reporter gene assay with human (h)ANP/luc and BNP/luc.

$R T-q P C R$. Total RNA was extracted from the cultured cells and tissues using TRIzol reagent (Invitrogen Life Technologies, Carlsbad, CA) and reverse transcribed into cDNA using the PrimeScript RT reagent kit (Takara Biotechnology, Dalian, China), according to the manufacturer's instructions. The mRNA levels of target genes were quantified using SYBR Green Master mix (Takara Biotechnology) using an ABI Prism 7900 Sequence Detector system (Applied Biosystems, Foster City, CA). Each reaction was performed in duplicate, and changes in the relative gene expression normalized to levels of 18s RNA were determined using the relative threshold cycle method (21).
Immunohistochemistry. The myocardial sections (5- $\mu \mathrm{m}$ sections of cryostat frozen tissue) from the mice were stained with mouse monoclonal anti-8-hydroxy-2'-deoxyguanosine (8-OHdG) antibody (1:200; clone N45.1; eBioscience) to evaluate the degree of DNA damage in the heart. The staining was visualized by treatment with a solution of 3,3'-diaminobenzidine (Dako Cytomation Liquid DAB Substrate Chromogen System; Dako Japan, Tokyo, Japan) for $40 \mathrm{sec}$ at $4^{\circ} \mathrm{C}$. The 8 -OHdG-positive area was measured (five random fields to yield $\sim 400$ cardiomyocytes) using Image $\mathbf{J}$ software version 1.46 (National Institutes of Health, Bethesda, MD, USA). An Olympus optical microscope was used (Axio Lab.A1 MAT; Olympus, Tokyo, Japan).

Western blotting. The protein levels of netrin-1, phosphorylated (p-)ERK1/2, p-MEK1/2, p-JNK1/2 and p-P38 were determined by western blot analysis. The protein extracted from the cells or tissues was separated on 10\% SDS-polyacrylamide electrophoresis gels (Sigma-Aldrich) and transferred onto nitrocellulose membranes (Pierce Biotechnology, Rockford, IL, USA). Following being blocked with 5\% non-fat milk in Tris-buffered saline for $3 \mathrm{~h}$, the membranes were incubated with the indicated primary antibodies $(0.2 \mu \mathrm{g} / \mathrm{ml}$; rabbit polyclonal ERK1/2 antibody, cat. no. 16443-1-AP, Proteintech, Chicago, IL, USA; rabbit anti-phospho-JNK 1/2, cat. no. Rs-1640R, Sigma-Aldrich; rabbit monoclonal MEK1 antibody, cat. no. AJ1468a, Abgent, San Diego, CA, USA; rabbit polyclonal p38 antibody, cat. no. NB100-56665, Novus Biologicals, Littleton, CO, USA) at $4^{\circ} \mathrm{C}$ overnight, followed by incubation with horseradish peroxidase-conjugated goat anti-rabbit $\operatorname{IgG}$ secondary antibody (1:5,000; eBioscience) for $3 \mathrm{~h}$. All the lanes were probed for $\beta$-actin as loading controls and the proteins were detected using an enhanced chemiluminescence detection kit (GE Healthcare Bio-Sciences, Pittsburgh, PA, USA).

Statistical analysis. Data are presented as the mean \pm standard error of the mean. Differences between the groups were evaluated using one-way analysis of variance with Bonferroni's post-hoc test. Survival curves, following TAC, were generated using the Kaplan-Meier method and were then compared using the log-rank test. $\mathrm{P}<0.05$ was considered to indicate a statistically significant difference. The statistical analyses were performed using the standard statistical software program JMP version 8 (SAS Institute Inc., Cary, NC, USA).

\section{Results}

Expression of netrin-1 decreases in murine hearts following $T A C$. To investigate the expression of netrin-1 during LV remodeling, the gene and protein levels of netrin- 1 was investigated in heart samples from mice following pressure overload, generated by TAC. It was found that the expression of netrin-1 was markedly decreased following TAC (Fig. 1A and B). Furthermore, the expression of netrin-1 was examined in neonatal rat cardiomyocytes following ET-1 stimulation. Similar to the observations in the heart samples from the TAC mice, the expression of netrin-1 was suppressed following ET-1 stimulation (Fig. 1C).

Netrin-1 suppresses the expression of cardiac foetal gene. To determine the role of netrin-1 on cardiac hypertrophy, 
A

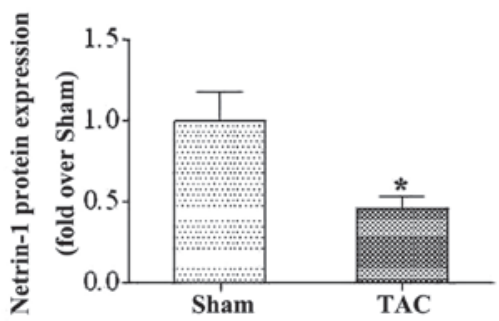

C

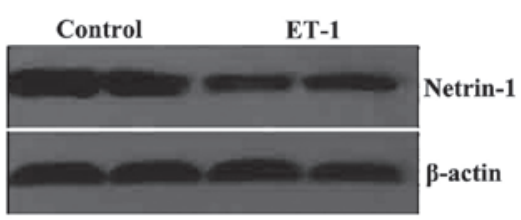

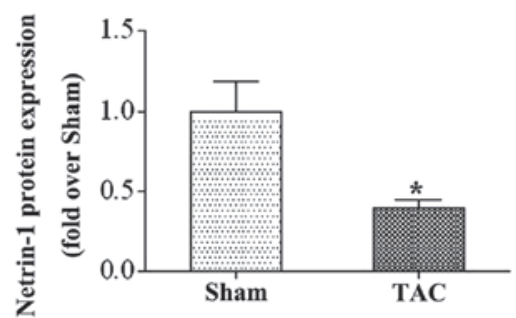

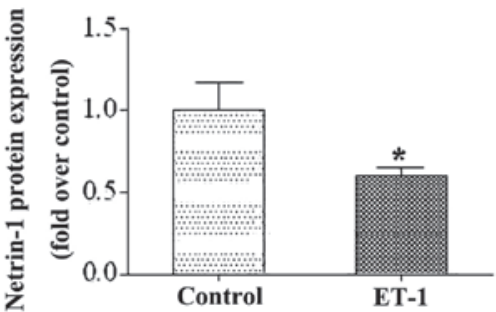

Figure 1. Expression of netrin-1 decreases in murine hearts following TAC. (A) mRNA expression of netrin-1 was analyzed by reverse transcription-quantitative polmyerase chain reaction. (B) Protein expression of netrin-1 was analyzed by western blotting. (C) Expression of netrin-1 in neonatal rat cardiomyocytes following ET-1 (100 nM) stimulation. Data are expressed as the mean \pm standard error of the mean. ${ }^{*} \mathrm{P}<0.05$, vs. all other groups $(\mathrm{n}=6)$. TAC, transverse aortic constriction; ET-1; endothelin-1; Sham, no TAC; Control, unstimulated.

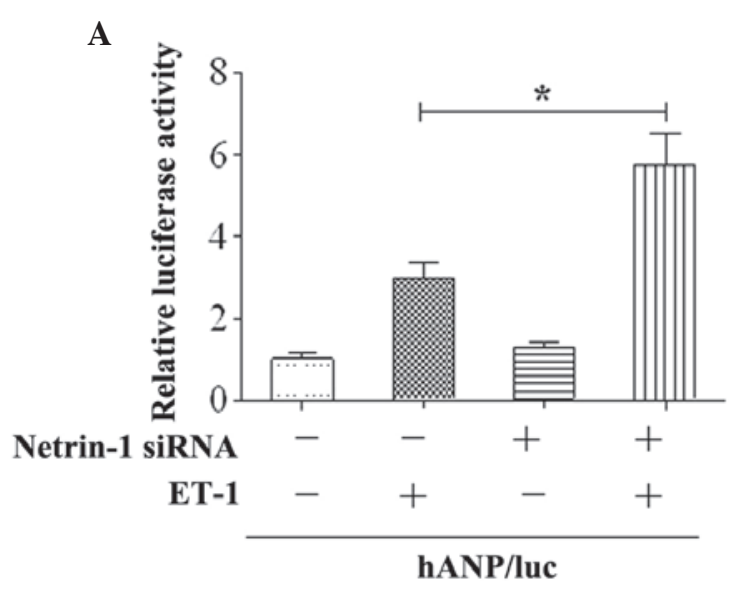

B

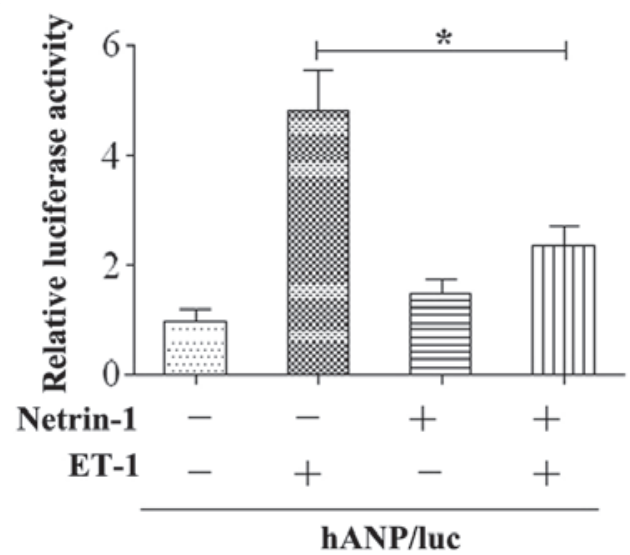

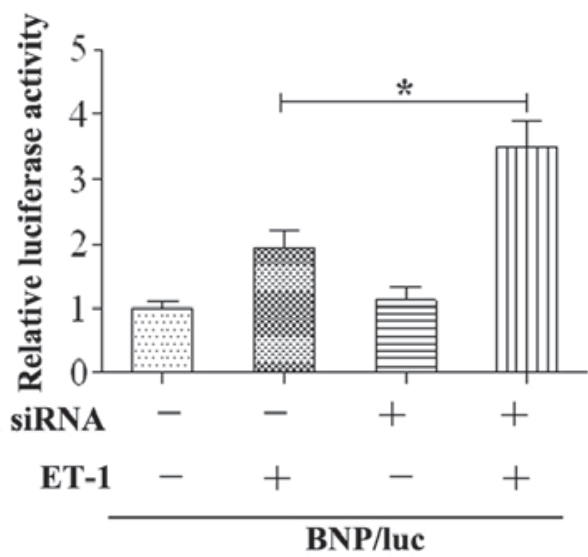

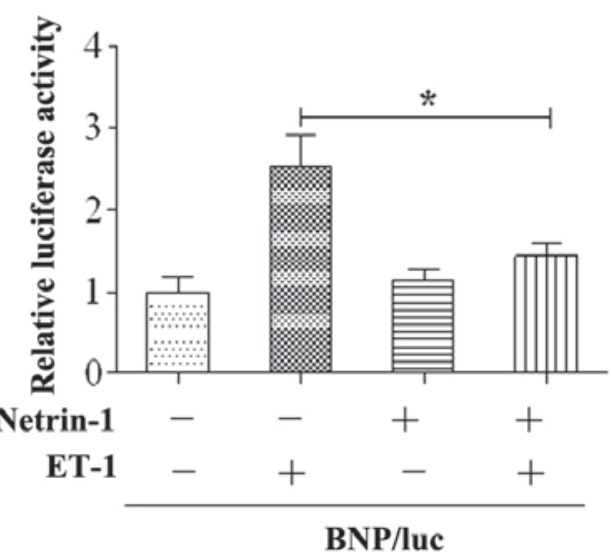

Figure 2. Netrin-1 suppresses the expression of cardiac foetal genes. (A) Increase in ANP and BNP promoter activity by netrin-1 siRNA transfection following ET-1 stimulation. (B) Suppressed ANP and BNP promoter activities following ET-1 stimulation in netrin-1 vector-transfected cardiomyocytes. Data are expressed as the mean \pm standard error of the mean. ${ }^{*} \mathrm{P}<0.05$, vs. all other groups $(\mathrm{n}=6)$. ET-1, endothelin-1; h, human; ANP, A-type natriuretic peptide; BNP, B-type natriuretic peptide; luc, luciferase.

the expression of foetal cardiac gene was examined in vitro. The results revealed that the activities of the ANP and BNP promoters were increased by ET-1 stimulation in the cardiomyocytes, and co-transfection with netrin-1 siRNA enhanced the activities of the ANP and BNP promoters (Fig. 2A). Subsequently, netrin-1 was co-transfected with the 

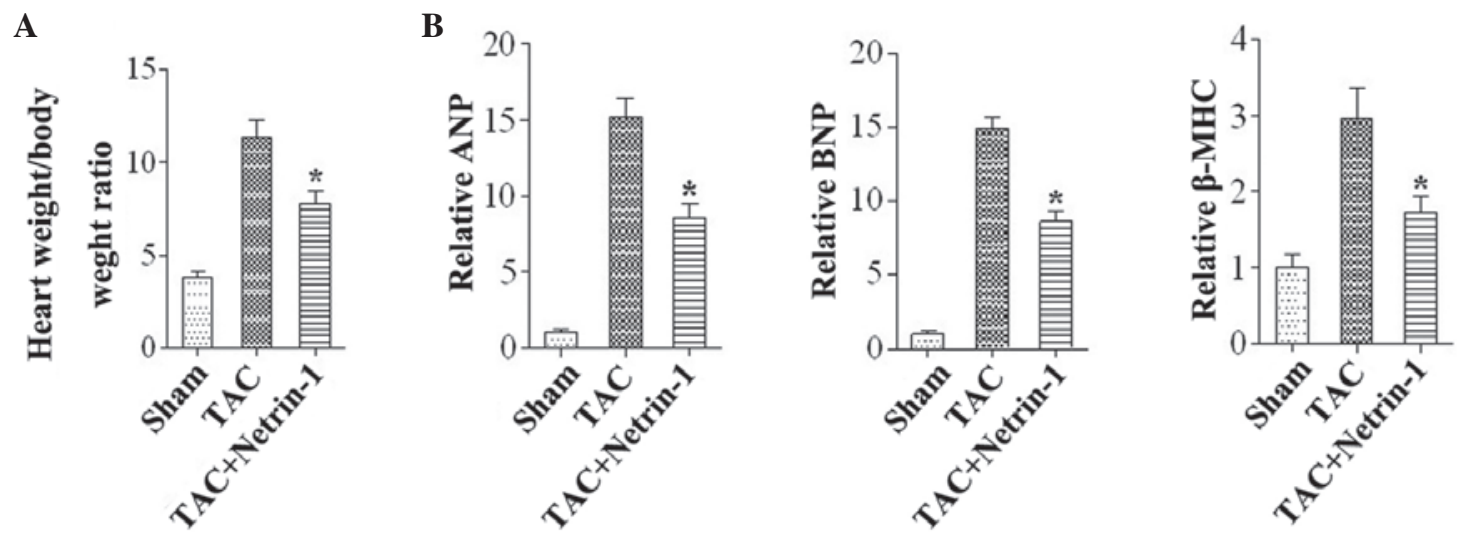

C

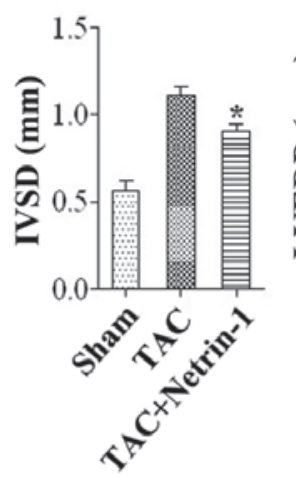

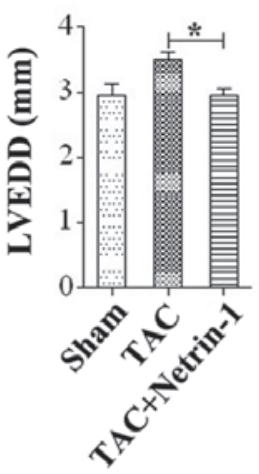
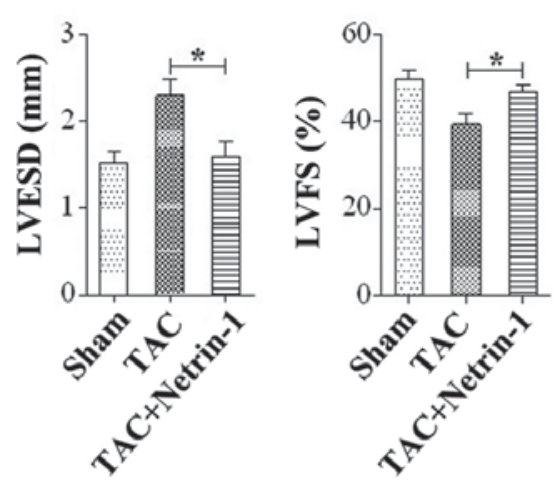

D

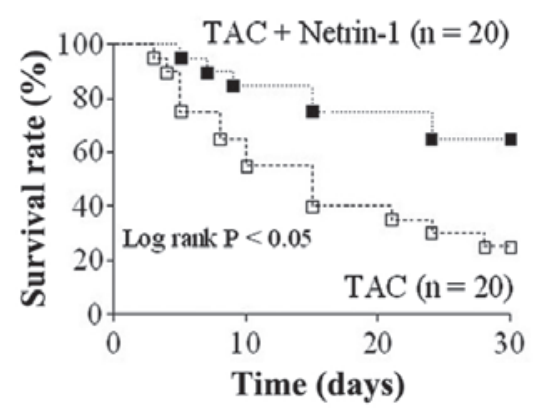

Figure 3. Netrin-1 attenuates the development of cardiac hypertrophy and heart failing. (A) Heart weight: body weight ratios in the TAC and sham group mice. (B) Quantitative analysis of the gene expression levels of ANP, BNP and $\beta$-MHC in mice from the Sham, TAC and TAC+Netrin- 1 groups. * $<0.05$, vs. Sham and TAC $(n=6)$. (C) Data from the echocardiographic measurements in mice from the Sham, TAC and TAC + Netrin-1 groups. ${ }^{*}<0.05$, vs. Sham and TAC for IVSD; * $<<0.05$, vs TAC group for LVEDD, LVESD and \%LVFS ( $\mathrm{n}=6$ ). Data are expressed as the mean \pm standard error of the mean. (D) Survival curves of mice in the TAC and TAC + netrin-1 groups. IVSD, interventricular wall thickness; LVEDD, left-ventricular end-diastolic dimension; LVESD, left-ventricular end-systolic dimension;\%LVFS, left-ventricular fractional shortening; TAC, transverse aortic constriction; Sham, no TAC; MHC, major histocompatibility complex; Sham, no TAC; ANP, A-type natriuretic peptide; BNP, B-type natriuretic peptide.

ANP/luc or BNP/luc constructs. The results demonstrated that overexpression of netrin-1 significantly attenuated the activities of the ANP and BNP promoters following ET-1 stimulation (Fig. 2B).

Netrin-1 attenuates the development of cardiac hypertrophy and heart failure. To examine the role of netrin-1 in the development of cardiac hypertrophy and heart failure in vivo, mice were subjected to either TAC or sham surgery. At 4 weeks following the TAC surgery, the increase in the weight of the hearts was significantly lower in Uthe mice treated with netrin-1 (Fig. 3A). The expression levels of ANP, BNP and $\beta$-MHC were significantly upregulated in the TAC group compared with the sham group, and this increase was markedly attenuated in the TAC mice treated with netrin-1 (Fig. 3B). Furthermore, systolic dysfunction and left ventricular dilation subsequent to TAC were attenuated in mice administered with netrin-1 compared with the control (Fig. 3C). The survival rate following TAC was significantly higher in mice treated with netrin-1 compared with those without netrin-1 treatment (Fig. 3D).

To investigate the role of netrin-1 in protecting cardiomyocytes from DNA damage in cardiac hypertrophy, immunohistochemical staining of the hearts from the TAC group were performed using anti-8-OHdG antibody. The mice in the sham group did not exhibit $8-\mathrm{OHdG}$-positive cardiomyocytes. In the TAC mice, the expression of $8-\mathrm{OHdG}$ was significantly increased, whereas the induction of 8-OHdG was suppressed in the netrin-1-treated mice (Fig. 4).

Netrin-1 inhibits the pressure overload-mediated MEK-ERK1/2 and JNK1/2 signaling pathways. The results of the present study demonstrated that netrin-1 has a protective role in cardiac hypertrophy. However, the molecular mechanisms through which netrin-1 treatment affects the hypertrophic responses on stress stimuli remain to be elucidated. To determine these possible mechanisms, the present study investigated the expression and activity of the MAPK signaling molecules, MEK1/2, ERK1/2, JNK1/2 and P38, as the MAPK pathway is known to be involved in pathological cardiac hypertrophy (22). The expression levels of p-MEK1/2, ERK1/2, and JNK1/2 were markedly increased in the TAC group compared with the sham group, and this increase was markedly attenuated in the TAC mice treated with netrin-1 (Fig. 5).

\section{Discussion}

The present study demonstrated the importance of netrin-1 in the development of cardiac hypertrophy and heart failure. The 


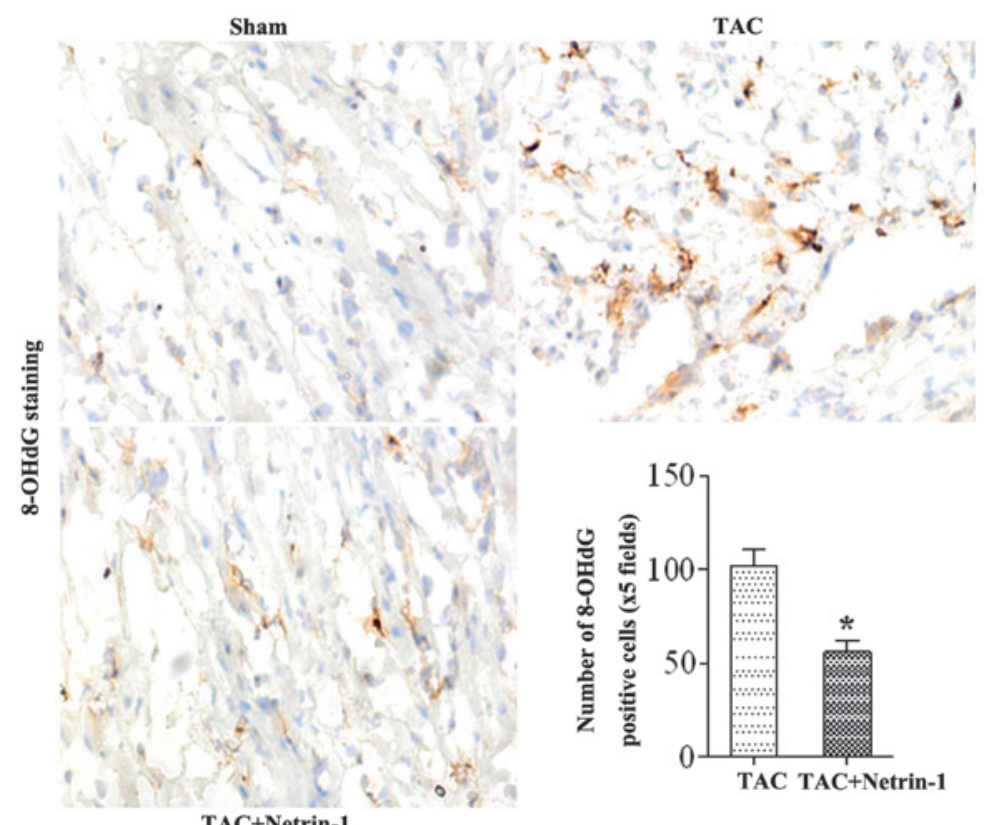

Figure 4. Immunohistochemical staining of heart tissue with anti-8-OHdG antibody 4 weeks after TAC. Sham-operated mice did not show 8-OHdG positive cardiomyocytes. In addition, in mice undergoing TAC, 8-OHdG expression was significantly increased, whereas the induction of 8-OHdG was suppressed in netrin-1-treated mice. 8-OHdG staining; magnification, $\mathrm{x} 100$. ${ }^{*} \mathrm{P}<0.05$, vs. TAC $(\mathrm{n}=6)$. TAC, transverse aortic constriction; Sham, no TAC; 8-OHdG, 8-hydroxy-2'-deoxyguanosine.

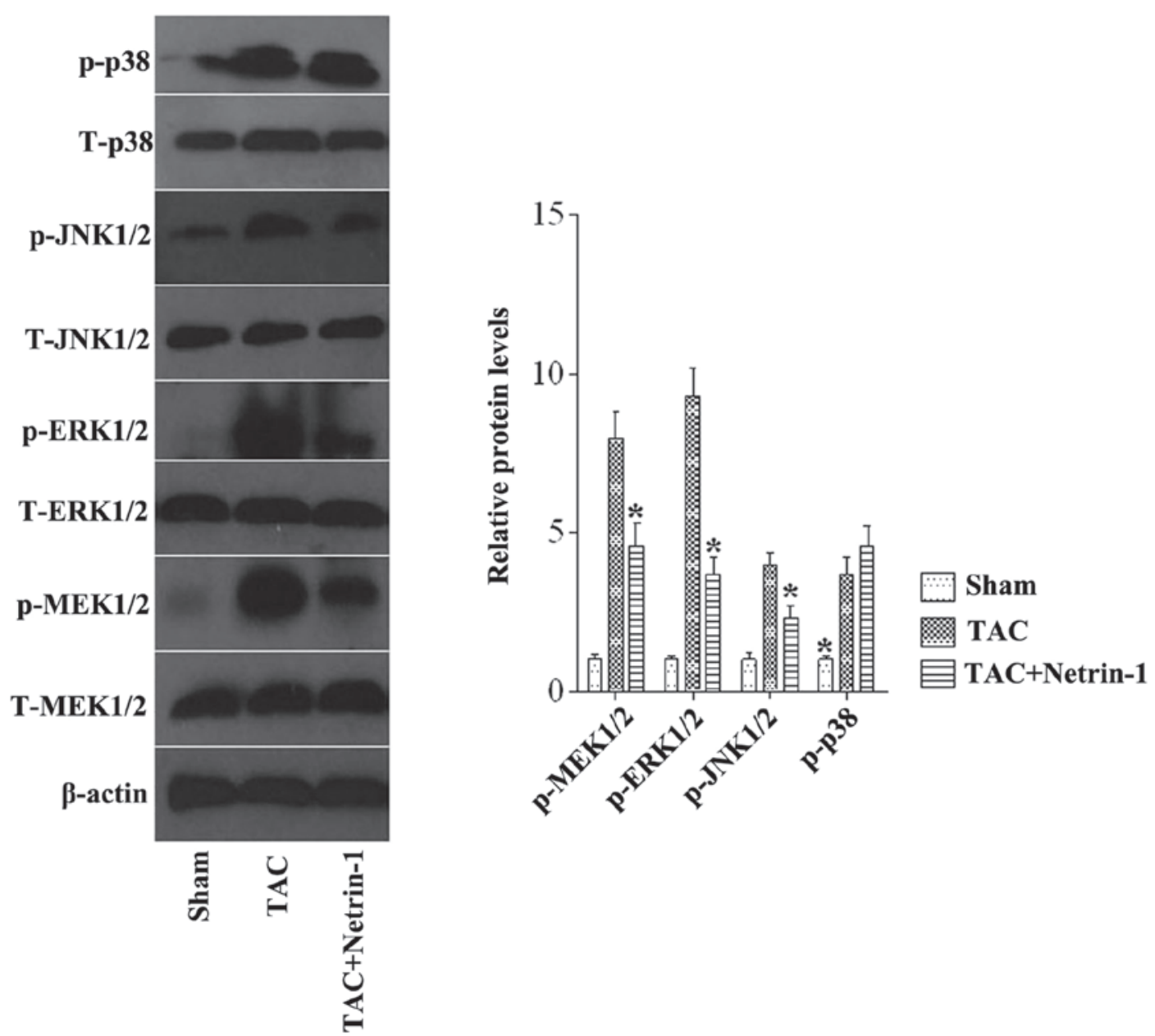

Figure 5. Representative western blots and quantitative results of the phosphorylated and total protein levels of MEK1/2, ERK1/2, JNK1/2 and P38 in mice from the Sham, TAC, and TAC + netrin-1 groups. "P<0.05 vs. all other groups ( $\mathrm{n}=6)$. Data are expressed as the mean \pm standard error of the mean. TAC, transverse aortic constriction; Sham, no TAC; p-, phosphorylated; MEK, mitogen-activated protein kinase kinase; ERK, extracellular-regulated protein kinase; JNK, c-Jun N-terminal kinase. 
results revealed that the expression of netrin-1 was decreased in TAC mice and neonatal rat cardiomyocytes in response to ET-1 stimulation. The results also demonstrated that the loss of netrin-1 aggravated the expression of foetal genes induced by the ET-1 stimulation. By contrast, this increase in gene expression levels was suppressed by treatment with netrin-1, and netrin-1 eliminated ventricular remodeling, cardiac dysfunction and DNA damage during pressure overload. Furthermore, the analyses of the signaling events indicated that the netrin-1-mediated protection against cardiac hypertrophy was attributed to interrupting the MEKK1-dependent MEK-ERK1/2 and JNK1/2 signaling pathways.

Netrin-1 is a laminin-associated molecule and is secreted at the spinal cord midline, where it is involved in guiding vertebrate commissural axons. A previous study reported that netrin-1 is expressed by the vascular endothelium, particularly in post capillary venules (23). The present study demonstrated that the expression of foetal genes induced by ET-1 was inhibited by the overexpression of netrin-1. In addition, the pressure overload-induced expression of foetal genes is attenuated following treatment of netrin-1 compared with control. Pressure overload-induced oxidative stress contributes to cardiac DNA damage and DNA repair/synthesis in failing hearts with systolic dysfunction (24). Therefore, DNA damage is considered to be a key pathogenic factor in ventricular dysfunction (25). A previous study demonstrated the involvement of netrin-1 in protecting against DNA-damage (26). In the present study, $8-\mathrm{OHdG}$ induction following TAC was significantly attenuated in the netrin-1-treated mice compared with control. Taken together, these findings indicated that netrin-1 may prevent DNA damage during pressure overload, as observed in the decrease in cardiac dysfunction in mice treated with netrin-1. A decrease in netrin-1 induced by hypertrophic stimulation may cause DNA damage, increasing the severity of cardiac hypertrophy, affecting the expression of foetal genes and causing cardiac dysfunction.

The mechanism underlying the antihypertrophic effect of netrin-1 remains to be fully elucidated. Considerable evidence exists to indicate that activation of the MAPK signaling pathway contributes to the pathogenesis of cardiac hypertrophy (27). MAPK signaling is mediated through three-kinase cascades of associated protein isoforms, beginning with an upstream MAPKKK (MEKK) and leading sequentially to MEK and effector MAPK. MAPK pathways have been implicated in a wide array of cellular responses to environmental stimuli $(28,29)$. Extensive evidence has documented the involvement of subdivisions of the ERK, JNK and p38 MAPKs in specific aspects of cardiac remodeling $(30,31)$. To clarify the molecular mechanisms involved in the suppressive effect of netrin-1 in cardiac hypertrophy, the present study evaluated the activation status of the MAPK pathway in hypertrophic models. The results demonstrated that the activation of MEK1/2, ERK1/2 and JNK1/2 was significantly enhanced in response to chronic pressure overload, and this upregulation was markedly inhibited by netrin- 1 . However, netrin-1 administration did not effect the protein expression of P38 in the hypertrophic models. Therefore, it is conceivable that netrin-1 exerted its antihypertrophic effects through inhibition of MEK-ERK1/2 and JNK1/2 signaling.
In conclusion, the present study provided evidence to support the hypothesis that netrin-1 protects against pressure overload-induced cardiac hypertrophy and heart failure through negative regulation of the MEKK1-dependent MEK-ERK1/2 and JNK1/2 signaling pathways. These findings suggest that netrin-1 may be a novel therapeutic target for the prevention of pathological cardiac hypertrophy.

\section{References}

1. McMurray JJ and Pfeffer MA: Heart failure. Lancet 365: 1877-1889, 2005.

2. Roger VL, Weston SA, Redfield MM, et al: Trends in heart failure incidence and survival in a community-based population. JAMA 292: 344-350, 2004.

3. Tessier-Lavigne $\mathrm{M}$ and Goodman CS: The molecular biology of axon guidance. Science 274: 1123-1133, 1996.

4. Barallobre MJ, Pascual M, Del RJ and Soriano E: The Netrin family of guidance factors: emphasis on Netrin-1 signalling. Brain Res Brain Res Rev 49: 22-47, 2005.

5. Tadagavadi RK, Wang W and Ramesh G: Netrin-1 regulates Th1/Th2/Th17 cytokine production and inflammation through UNC5B receptor and protects kidney against ischemia-reperfusion injury. J Immunol 185: 3750-3758, 2010.

6. Rosenberger P, Schwab JM, Mirakaj V, et al: Hypoxia-inducible factor-dependent induction of netrin-1 dampens inflammation caused by hypoxia. Nat Immunol 10: 195-202, 2009.

7. Wang W, Reeves WB, Pays L, Mehlen P and Ramesh G: Netrin-1 overexpression protects kidney from ischemia reperfusion injury by suppressing apoptosis. Am J Pathol 175: 1010-1018, 2009.

8. Ly NP, Komatsuzaki K, Fraser IP, et al: Netrin-1 inhibits leukocyte migration in vitro and in vivo. Proc Natl Acad Sci USA 102: 14729-14734, 2005.

9. Mirakaj V, Thix CA, Laucher S, et al: Netrin-1 dampens pulmonary inflammation during acute lung injury. Am J Respir Crit Care Med 181:815-824, 2010.

10. Mirakaj V, Gatidou D, Potzsch C, Konig K and Rosenberger P: Netrin-1 signaling dampens inflammatory peritonitis. J Immunol 186: 549-555, 2011.

11. Rosenberger P, Schwab JM, Mirakaj V, et al: Hypoxia-inducible factor-dependent induction of netrin-1 dampens inflammation caused by hypoxia. Nat Immunol 10: 195-202, 2009.

12. Grenz A, Dalton JH, Bauerle JD, et al: Partial netrin-1 deficiency aggravates acute kidney injury. PLoS One 6: e14812, 2011.

13. Aherne CM, Collins CB, Masterson JC, et al: Neuronal guidance molecule netrin-1 attenuates inflammatory cell trafficking during acute experimental colitis. Gut 61: 695-705, 2012.

14. Zhang J and Cai H: Netrin-1 prevents ischemia/reperfusion-induced myocardial infarction via a DCC/ERK1/2/eNOS s1177/NO/DCC feed-forward mechanism. J Mol Cell Cardiol 48: 1060-1070, 2010

15. Durrani S, Haider KH, Ahmed RP, Jiang S and Ashraf M: Cytoprotective and proangiogenic activity of ex-vivo netrin-1 transgene overexpression protects the heart against ischemia/reperfusion injury. Stem Cells Dev 21: 1769-1778, 2012.

16. Ahmed RP, Haider KH, Shujia J, Afzal MR and Ashraf M: Sonic Hedgehog gene delivery to the rodent heart promotes angiogenesis via iNOS/netrin-1/PKC pathway. PLoS One 5: e8576, 2010.

17. Joseph BB and Quan PD: The neuroimmune guidance cue netrin-1: a new therapeutic target in cardiovascular disease. Am J Cardiovasc Dis 3: 129-134, 2013.

18. Shishido T, Woo CH, Ding B, et al: Effects of MEK5/ERK5 association on small ubiquitin-related modification of ERK5: implications for diabetic ventricular dysfunction after myocardial infarction. Circ Res 102: 1416-1425, 2008.

19. Funayama A, Shishido T, Netsu S, et al: Cardiac nuclear high mobility group box 1 prevents the development of cardiac hypertrophy and heart failure. Cardiovasc Res 99: 657-664, 2013.

20. Le NT, Takei Y, Shishido T, et al: p90RSK targets the ERK5-CHIP ubiquitin E3 ligase activity in diabetic hearts and promotes cardiac apoptosis and dysfunction. Circ Res 110: 536-550, 2012.

21. Jones ME, Mayne GC, Wang T, Watson DI and Hussey DJ: A fixed-point algorithm for estimating amplification efficiency from a polymerase chain reaction dilution series. BMC Bioinformatics 15: 372, 2014. 
22. Li H, Tang QZ, Liu C, et al: Cellular FLICE-inhibitory protein protects against cardiac remodeling induced by angiotensin II in mice. Hypertension 56: 1109-1117, 2010.

23. Ly NP, Komatsuzaki K, Fraser IP, et al: Netrin-1 inhibits leukocyte migration in vitro and in vivo. Proc Natl Acad Sci USA 102: 14729-14734, 2005.

24. Siggens L, Figg N, Bennett M and Foo R: Nutrient deprivation regulates DNA damage repair in cardiomyocytes via loss of the base-excision repair enzyme OGG1. FASEB J 26: 2117-2124, 2012.

25. SuzukiS,ShishidoT,IshinoM,etal:8-Hydroxy-2'-deoxyguanosine is a prognostic mediator for cardiac event. Eur J Clin Invest 41 : 759-766, 2011.

26. Wang H, Ozaki T, Shamim HM, et al: A newly identified dependence receptor UNC5H4 is induced during DNA damage-mediated apoptosis and transcriptional target of tumor suppressor p53. Biochem Biophys Res Commun 370: 594-598, 2008.
27. Van Berlo JH, Maillet M and Molkentin JD: Signaling effectors underlying pathologic growth and remodeling of the heart. J Clin Invest 123: 37-45, 2013.

28. Garrington TP and Johnson GL: Organization and regulation of mitogen-activated protein kinase signaling pathways. Curr Opin Cell Biol 11: 211-218, 1999.

29. Kuida K and Boucher DM: Functions of MAP kinases: insights from gene-targeting studies. J Biochem 135: 653-656, 2004.

30. Ravingerova T, Barancik M and Strniskova M: Mitogen-activated protein kinases: a new therapeutic target in cardiac pathology. Mol Cell Biochem 247: 127-138, 2003.

31. Wang Y: Mitogen-activated protein kinases in heart development and diseases. Circulation 116: 1413-1423, 2007. 